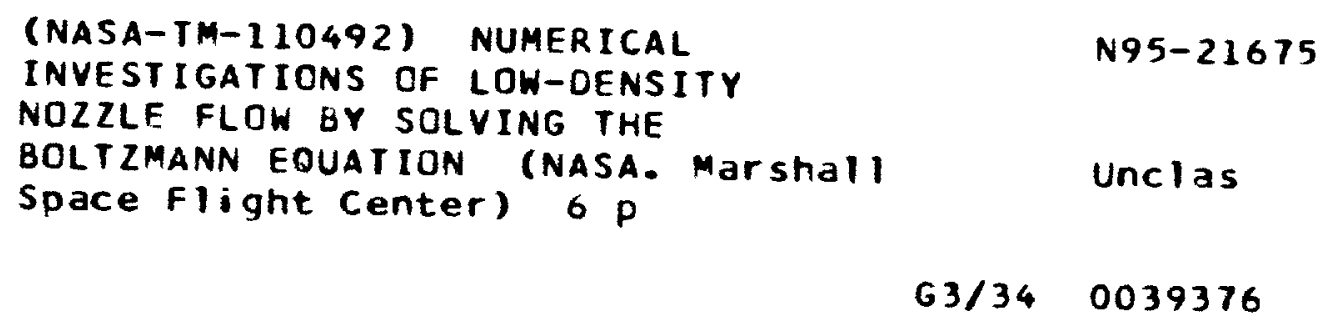

Unclas

$63 / 34 \quad 0039376$ 


\title{
NUMERICAL INVESTIGATIONS OF LOW-DENSITY NOZZLE FLOW BY SOLVING THE BOLTZMANN EQUATION
}

\author{
Zheng-Tao Deng ${ }^{\dagger}$, Goang-Shin Liaw ${ }^{\ddagger}$ \\ Department of Civil Engineering \\ Alabama AEM University \\ Huntsville, Alabama 35762 \\ Lynn Chen Chou* \\ Structure and System Dynamics Labs / Fluid Dynamics Division / ED 93 \\ NASA/Marshall Space Flight Center \\ Huntsville, AL 35812
}

\begin{abstract}
A two-dimensional finite-difference code to solve the BGK-Boltzmann equation has been developed. The solution procedure consists of three steps: (1). Transforming the BGK-Boltzmann equation into two simultaneous partial differential equations by taking moments of the distribution function with respect to the molecular velocity $u_{z}$, with weighting factors 1 and $u_{z}^{2}$. (2). Solving the transformed equations in the physical space based on the time-marching technique and the four-stage RungeKutta time integration, for a given discrete-ordinate. The Roe's second-order upwind difference scheme is used to discretize the convective terms and the collision terms are treated as source terms. (3). Using the newly calculated distribution functions at each point in the physical space to calculate the macroscopic flow parameters by the Modified Gaussian quadrature formula. Repeating Steps 2 and 3 , the time-marching procedure stops when the convergent criteria is reached. A low-density nozzle flow field has been calculated by this newly developed code. The BGK Boltzmann solution and the experimental data show excellent agreement. It demonstrated that numerical solutions of the BGK-Boltzmann equation are ready to be experimentally validated.
\end{abstract}

\section{INTRODUCTION}

Low density nozzles are often used as a propulsive system for the trajectory control of satellites and other spacecrafts in orbit. One of the distinct characteristics of the low-density nozzle flow is that the expanding gas is highly rarefied with a thick boundary layer near the wall such that the distinction between the boundary layer and

$\dagger$ Assistant Professor, Member AIAA

$\ddagger$ Professor, Member AIAA

* AST, Senior Member AIAA

Copyright (C)American Institute of Aeronautics and Astronautics. Inc.. 1995. All rights reserved. the core flow disappears. Thus the fluid dynamics in a low density nozzle falls into the transitional regime. It has been well known that the conventional Navier-Stokes equations fail to predict flow characteristics accurately in the transitional flow regime ${ }^{[1,2]}$. Usage of improper continuum methods will lead to significant erroneous estimates of the nozzle flow characteristics ${ }^{[3]}$. A rigorous numerical treatment of the flow field within the low-density nozzle requires the solution of the Boltzmann equation.

The Boltzmann equation is a highly non-linear integro-differential equation to describe flow characteristics in the molecular level with the molecular distribution function as the only dependent variable. Direct numerical solutions of the Boltzmann equation for fluid flows involves the calculation of the molecular distribution function as well as the collision integral at each velocity point in a three-dimensional infinite velocity space ${ }^{[4]}$.

Many research efforts have been reported in directly solving the non-linear Boltzmann equation using finite-difference methods. The Monte-Carlo-Integration technique $(\mathrm{MCI})^{[5,6, \pi]}$ has been exclusively used to evaluate the collision integrals. The shortcoming of the $\mathrm{MCI}$ method is the large consumption of computer time, and the MCI procedure must be executed at each time step. Without loss of generality, the linearized BGKBoltzmann equation has been solved successfully with large Knudsen number ${ }^{[8,9]}$. The advantage of solving the BGK-Boltzmann equation is that it requires much less computational efforts and computer time, and still preserves the flow characteristics of the original Boltzmann equation, as long as the Knudsen number is relatively large. For engineering applications, the solutions of the macroscopic parameters are required. These parameters are derived from the molecular distribution function. The discrete-ordinate method ${ }^{[8.9]}$ is based on the concept that the integration over an infinite velocity space can be reduced to integration over a finite number of discrete points by selecting a appropriate integration formula. The transformed finite difference equations from the Boltzmann equation can be quantitized in the velocity space, and the macroscopic properties can be obtained though this quantitized velocity space.

The objective of this research is to develop a new finite-difference method to solve the BGK-Boltzmann equation for nozzle flows in the transitional flow regime. 
The new methodology combines the Discrete-Ordinate (FDDO) technique ${ }^{[9]}$ with a second-order upwind finitedifference method ${ }^{[10]}$ and the four-stage Runge-Kutta time integration scheme. This paper describes the numerical procedure and presents solutions of low-density nozzle flows.

\section{MATHEMATICAL FORMULATION}

A two-dimensional BGK-Boltzmann equation, without external force, is solved in a Cartesian coordinate system

$$
\frac{\partial f}{\partial t}+u_{x} \frac{\partial f}{\partial x}+u_{y} \frac{\partial f}{\partial y}=A_{c}\left(f_{0}-f\right)
$$

where $f_{0}$ is the Maxwellian distribution function, $A_{c}$ is the collision frequency, and

$$
\begin{gathered}
f_{0}=\frac{n}{(2 \pi R T)^{\frac{3}{2}}} \operatorname{Exp}\left(\left(-\frac{c^{2}}{2 R T}\right)\right) \\
A_{c}=\frac{m n R T}{\mu}
\end{gathered}
$$

$m$ is the molecular weight, $n$ is the molecular number density, $u_{x}, u_{y}$ are the molecular velocity components in the $x$ - and $y$-directions, respectively. It is assumed that the viscosity coefficient $\mu$ and molecular mean free path $\lambda_{1}$ are

$$
\begin{gathered}
\frac{\mu}{\mu_{0}}=\left(\frac{T}{T_{0}}\right)^{\omega} \\
\lambda_{1}=\frac{16}{5} \frac{\mu}{m n_{0}(2 \pi R T)^{\frac{1}{2}}}
\end{gathered}
$$

The subscript 0 indicates the reference state, and the exponent $\omega$ depends on the flow media. Equation (1) is simplified as

$$
\begin{gathered}
\frac{\partial f}{\partial t}+u_{x} \frac{\partial f}{\partial x}+u_{y} \frac{\partial f}{\partial y}=\frac{16}{5} \frac{1}{\lambda_{1}} \frac{n}{n_{0}}\left(\frac{R T}{2 \pi}\right)^{\frac{1}{2}} \cdot\left(\frac{T_{0}}{T}\right)^{\omega} \\
\cdot\left(\frac{n}{(2 \pi R T)^{\frac{3}{2}}} \operatorname{Exp}\left(-\frac{c^{2}}{2 R T}\right)-f\right)
\end{gathered}
$$

In order to reduce the number of independent variables, the $\mathrm{BGK}$ Boltzmann equation is integrated with respect to $u_{z}$, with weighting factors 1 and $u_{z}^{2}$. Equation (1) becomes

$$
\begin{aligned}
& \frac{\partial g}{\partial t}+u_{x} \frac{\partial g}{\partial x}+u_{y} \frac{\partial g}{\partial y}=A_{c}(G-g) \\
& \frac{\partial h}{\partial t}+u_{x} \frac{\partial h}{\partial x}+u_{y} \frac{\partial h}{\partial y}=A_{c}(H-h)
\end{aligned}
$$

where

$$
\begin{gathered}
g=g\left(x, y, u_{x}, u_{y}\right)=\int_{-\infty}^{+\infty} f\left(x, y, u_{x}, u_{z}, u_{z}\right) d u_{z} \\
h=h\left(x, y, u_{x}, u_{y}\right)=\int_{-\infty}^{+\infty} u_{z}^{2} f\left(x, y, u_{x}, u_{z}, u_{z}\right) d u_{z} \\
G=G\left(x, y, u_{x}, u_{y}\right)=\int_{-\infty}^{+\infty} f_{0} d u_{z} \\
H=H\left(x, y, u_{x}, u_{y}\right)=\int_{-\infty}^{+\infty} u_{z}^{2} f_{-} d u_{z}
\end{gathered}
$$

Taking a one-one correspondence transformation in the velocity space as well as in the physical space

$$
\begin{gathered}
u_{x}=V \sin \phi \\
u_{y}=V \cos \phi \\
\phi=\tan ^{-1}\left(\frac{u_{x}}{u_{y}}\right)
\end{gathered}
$$

equations (7) and (8) become

$$
\begin{aligned}
& \frac{\partial g}{\partial \tau}+B \frac{\partial g}{\partial \eta}+C \frac{\partial g}{\partial \xi}=A_{c}(G-g) \\
& \frac{\partial h}{\partial \tau}+B \frac{\partial h}{\partial \eta}+C \frac{\partial h}{\partial \xi}=A_{c}(H-h)
\end{aligned}
$$

where

$$
\begin{aligned}
& B=\frac{1}{J}\left(V \cos \phi x_{\xi}-V \sin o y_{\xi}\right) \\
& C=\frac{1}{J}\left(V \sin \phi y_{\eta}-V \cos o x_{\eta}\right)
\end{aligned}
$$

with Jacobian $J(x, y ; \xi, \eta)$ and coordinate transformation coefficients $x_{\xi}, x_{\eta}, y_{\xi}, y_{\eta}$.

\section{NUMERICAL PROCEDURE}

The macroscopic flow parameters can be obtained through the integration of the distribution function $f$. For example, the number density, $N(x . y . t)$ in twodimensional cartesian coordinates can be obtained from the zero-moment equation.

$$
\begin{aligned}
& N(x, y, t)= \\
& \int_{-\infty}^{\infty} \int_{-\infty}^{\infty} \int_{-\infty}^{\infty} f\left(x, y, t, u_{x}, u_{y}, u_{z}\right) d u_{x} d u_{y} d u_{z}
\end{aligned}
$$

where $\left(u_{x}, u_{y}, u_{z}\right)$ is the molecular velocity vector in the velocity space.

Substituting equation (9) into equation (20), it becomes

$$
N(x, y, t)=\int_{-\infty}^{\infty} \int_{-\infty}^{\infty} g\left(x, y, t, u_{x}, u_{y}\right) d u_{z} d u_{y}
$$

Using polar coordinates for velocity space to rewrite equation (21), i.e.. 


$$
\begin{gathered}
u_{x}=I \sin \phi \\
u_{y}=I \cos \phi \\
0 \leq I_{m}, \quad 0 \leq 0 \leq 2 \pi
\end{gathered}
$$

Then, equation (21) is transformed into

$$
N(x, y, t)=\int_{0}^{\infty} \int_{0}^{2 \pi} V \sin \phi g(x, y, t, V, \phi) d \phi d V
$$

Since the molecular distribution function, $f$, is in an exponential form in nature, it is reasonable to assume that $g(x, y, t)$ is a function of the exponential type. Then. equations (24) can be rewritten as

$$
N(x, y, t)=\int_{0}^{\infty} e^{-V^{2}} V^{\omega} P(V) d V
$$

where

$$
P(V)=\int_{0}^{2 \pi} e^{+V^{2}} V^{1-\omega} g\left(x, y, t, V_{m}, \phi\right) d \phi
$$

Equation (25) can be integrated effectively using the modified Gauss-Hermite quadrature formula. This quadrature formula replaces the semi-infinite integral by a finite number of integration points, called "roots $V_{k}, \quad k=1,2, \cdots n "$, and transforms equation (25) into

$$
N(x, y, t)=\sum_{k=1}^{n} W_{k} P\left(V_{k}\right)
$$

where $W_{k}$ is the corresponding weighting factor for root $V_{k}$. For $\omega=1, n=16$, the root and its weighting factors are given as

$$
\begin{aligned}
& V_{1}=0.477579953723861 E-01 \\
& V_{2}=0.157564360925804 E+00 \\
& V_{3}=0.323655656470272 E+00 \\
& V_{4}=0.539147354111002 E+00 \\
& V_{5}=0.797005397275377 E+00 \\
& V_{6}=0.109095830650419 E+01 \\
& V_{7}=0.141597596974798 E+01 \\
& V_{5}=0.176843702942131 E+01 \\
& V_{9}=0.214614996091144 E+01 \\
& V_{10}=0.254836565149444 E+01 \\
& V_{11}=0.297589659136340 E+01 \\
& V_{12}=0.343148386715786 E+01 \\
& V_{13}=0.392069411852247 E+01 \\
& V_{14}=0.445412057238520 E+01 \\
& V_{15}=0.505367426854191 E+01 \\
& V_{16}=0.577847884687290 E+01
\end{aligned}
$$

$$
\begin{aligned}
& W_{1}=0.379530780467479 E-02 \\
& W_{2}=0.213680829692996 E-01 \\
& W_{3}=0.559585707892949 E-01 \\
& W_{4}=0.958716826650700 E-01 \\
& W_{5}=0.116908207001337 E+00 \\
& W_{6}=0.102936301287559 E+00 \\
& W_{7}=0.646824672793000 E-01 \\
& W_{5}=0.283191162204620 E-01 \\
& W_{9}=0.836264802590032 E-02 \\
& W_{10}=0.159773621113803 E-02 \\
& W_{11}=0.187013465916242 E-03 \\
& W_{12}=0.124393550561664 E-04 \\
& W_{13}=0.420846696187155 E-06 \\
& W_{14}=0.605184708943963 E-08 \\
& W_{15}=0.264340659193899 E-10 \\
& W_{16}=0.152459411718563 E-13
\end{aligned}
$$

and

$$
W_{k}=W_{k} e^{v_{k}^{2}}, k=1,2,3, \cdots, 16
$$

The root $V_{k}$ is called the Discrete-Ordinate.

For any selected discrete-ordinate $V_{k}$,

$$
\begin{gathered}
\frac{\partial g_{k}}{\partial \tau}+B_{k} \frac{\partial g_{k}}{\partial \eta}+C_{k} \frac{\partial g_{k}}{\partial \xi}=A_{c}\left(G_{k}-g_{k}\right) \\
\frac{\partial h_{k}}{\partial \tau}+B_{k} \frac{\partial h_{k}}{\partial \eta}+C_{k} \frac{\partial h_{k}}{\partial \xi}=A_{c}\left(H_{k}-h_{k}\right) \\
B_{k}=\frac{1}{J}\left(V_{k} \cos \phi x_{\xi}-V_{k} \sin \phi y_{\xi}\right) \\
C_{k}=\frac{1}{J}\left(V_{k} \sin \phi y_{\eta}-V_{k} \cos \phi x_{\eta}\right) \\
G_{k}=\int_{-\infty}^{+\infty} f_{0}\left(\xi, \eta, u_{k}, \phi, u_{z}\right) d u_{z} \\
H_{k}=\int_{-\infty}^{+\infty} u_{z}^{2} f_{0}\left(\xi, \eta, u_{k}, \phi, u_{z}\right) d u_{z} \\
(\mathbf{k}=\mathbf{1}, \mathbf{2}, 3, \ldots, \mathbf{N}-\mathbf{1}, \mathbf{N})
\end{gathered}
$$

In this study, a 16 point discrete-ordinate method is adopted. $N=16$.

Introduce the following dimensionless variables

$$
\begin{gathered}
\dot{x}=\frac{x}{d}, \quad \dot{y}=\frac{y}{d}, \quad \dot{n}=\frac{n}{n_{0}} \\
\dot{u}_{x}=\frac{u_{x}}{V_{0}}, \quad \dot{u}_{y}=\frac{u_{y}}{V_{0}} . \quad \dot{T}=\frac{T}{T_{0}} \\
\dot{A}_{c}=\frac{A_{c} d}{l_{0}}, \quad \dot{t}=\frac{t}{d / \sqrt{2 R T_{0}}} \\
\dot{g}=\frac{g l_{0}^{2}}{n_{0}} . \quad h=\frac{h}{n_{0}}
\end{gathered}
$$




$$
G=\frac{G l_{0}^{2}}{n_{0}}, \quad \dot{H}=\frac{H}{n_{0}}
$$

where $d$ is the characteristic length of the flow field, the subscript ' 0 ' represents the reference state, and

$$
V_{0}=\sqrt{2 R T_{0}}
$$

then, equations $(28,29)$ become

$$
\begin{aligned}
& \frac{\partial \hat{g}_{k}}{\partial \hat{\tau}}+\hat{B}_{k} \frac{\partial \hat{g}_{k}}{\partial \eta}+\dot{C}_{k} \frac{\partial \hat{g}_{k}}{\partial \xi}=\hat{A}_{c}\left(\hat{G}_{k}-\dot{g}_{k}\right) \\
& \frac{\partial \hat{h}_{k}}{\partial \hat{\tau}}+\hat{B}_{k} \frac{\partial \hat{h}_{k}}{\partial \eta}+\hat{C}_{k} \frac{\partial \hat{h}_{k}}{\partial \xi}=\hat{A}_{c}\left(\hat{H}_{k}-\hat{h}_{k}\right)
\end{aligned}
$$

A successful numerical scheme for solving equations (34) and (35) should be fast and reasonably accurate. An assessment of numerical techniques for solving these equations concludes that the explicit Runge-Kutta fourstage time-integration scheme with second order upwind flux differencing ${ }^{[10]}$ performed very well with regard to handling the nonlinearity and the convergence speed. Rewrite equations (34) and (35) into

$$
\frac{\partial Q}{\partial \tau}+\frac{\partial E}{\partial \eta}+\frac{\partial F}{\partial \xi}=S
$$

where $Q$ represents $g_{k}$ and $h_{k}$ in above equations, $E$ is $B_{k} Q, F$ is $C_{k} Q, S$ represents source terms. The fourstage Runge-Kutta integration technique is applied to solve equations (34) and (35). The solution procedure can be summarized as follows.

For a given discrete-ordinate $V_{k}$, equations (34) and (35) are solved, with $0 \leq \phi \leq 2 \pi$, for every points * in the physical space using the designated Runge-Kutta scheme. A characteristic-based spatial discretization using second-order upwind flux-differencing is adopted to discretize the convective terms. The explicit spatial operator may be written in terms of numerical flux vectors treated on a locally one-dimensional basis. The residue $R\left(Q_{i, j}\right)$ can be written as

$$
\begin{aligned}
R\left(Q_{i, j}\right)= & \frac{1}{\Delta \eta}\left(E_{i+\frac{1}{2}, j}^{(2)}-E_{i-\frac{1}{2}, j}^{(2)}\right) \\
& +\frac{1}{\Delta \xi}\left(F_{i, j+\frac{1}{2}}^{(2)}-F_{i, j-\frac{1}{2}}^{(2)}\right)-S_{i, j}
\end{aligned}
$$

The superscript (2) represents the second-order terms.

$$
\begin{aligned}
E_{i+\frac{1}{2}, j}^{(2)}=E_{i+\frac{1}{2}, j}^{(1)} & +\frac{1-\epsilon}{4}\left(\Delta \dot{E}_{i-\frac{1}{2}, j}^{+}-\Delta \dot{E}_{i+\frac{3}{2}, j}^{-}\right) \\
& +\frac{1+\epsilon}{4}\left(\Delta \dot{E}_{i+\frac{1}{2}, j}^{+}-\Delta \dot{E}_{i+\frac{1}{2}, j}^{-}\right) \\
F_{i, j+\frac{1}{2}}^{(2)}=F_{i, j+\frac{1}{2}}^{(1)} & +\frac{1-\epsilon}{4}\left(\Delta \hat{F}_{i, j-\frac{1}{2}, j}^{+}-\Delta \dot{F}_{i, j+\frac{3}{2}}^{-}\right) \\
+ & \frac{1+\epsilon}{4}\left(\Delta \hat{F}_{i, j+\frac{1}{2}, j}^{+}-\Delta \dot{F}_{i, j+\frac{1}{2}}^{-}\right)
\end{aligned}
$$

where

$$
\begin{aligned}
E_{i+\frac{1}{2}, j}^{(1)} & =\frac{1}{2}\left(E_{i, j}+E_{i+1, j}\right) \\
& -\frac{1}{2}\left(\left|B_{k}\right|_{i+\frac{1}{2}, j}\left(Q_{i+1, j}-Q_{i, j}\right)\right) \\
F_{i, j+\frac{1}{2}}^{(1)} & =\frac{1}{2}\left(F_{i, j}+F_{i, j+1}\right) \\
& -\frac{1}{2}\left(\left|C_{k}\right|_{i, j+\frac{1}{2}}\left(Q_{i, j+1}-Q_{i, j}\right)\right) \\
\Delta \hat{E}_{i+\frac{1}{2}, j}^{-} & =\operatorname{Minmod}\left(\Delta \sigma_{i+\frac{1}{2}, j}^{-}, \beta \Delta \sigma_{i-\frac{1}{2}, j}^{-}\right) \\
\Delta \dot{E}_{i+\frac{1}{2}, j}^{+} & =\operatorname{Minmod}\left(\Delta \sigma_{i+\frac{1}{2}, j}^{+}, \beta \Delta \sigma_{i+\frac{1}{2}, j}^{-}\right) \\
\Delta \dot{F}_{i, j+\frac{1}{2}}^{-} & =\operatorname{Minmod}\left(\Delta \sigma_{i, j+\frac{1}{2}}^{-}, \beta \Delta \sigma_{i, j-\frac{1}{2}}^{-}\right) \\
\Delta \hat{F}_{i, j+\frac{1}{2}}^{+} & =\operatorname{Minmod}\left(\Delta \sigma_{i, j+\frac{1}{2}}^{+}, \beta \Delta \sigma_{i, j-\frac{3}{2}}^{+}\right) \\
\Delta \sigma_{i+\frac{1}{2}, j}^{-} & =\frac{1}{2}\left(B_{k}-\left|B_{k}\right|\right)_{i+\frac{1}{2}, j}\left(Q_{i+1, j}-Q_{i, j}\right) \\
\Delta \sigma_{i+\frac{1}{2}, j}^{+} & =\frac{1}{2}\left(B_{k}+\left|B_{k}\right|\right)_{i+\frac{1}{2}, j}\left(Q_{i+1, j}-Q_{i, j}\right) \\
\Delta \sigma_{i, j+\frac{1}{2}}^{-} & =\frac{1}{2}\left(C_{k}-\left|C_{k}\right|\right)_{i, j+\frac{1}{2}}\left(Q_{i, j+1}-Q_{i, j}\right) \\
\Delta \sigma_{i, j+\frac{1}{2}}^{+} & =\frac{1}{2}\left(C_{k}+\left|C_{k}\right|\right)_{i+\frac{1}{2}, j}\left(Q_{i, j+1}-Q_{i, j}\right)
\end{aligned}
$$

and the Minmod operator is defined by

$$
\operatorname{Minmod}[x, y]=\operatorname{sgn}(x) \operatorname{Max}\left(0, \operatorname{Min}\left\{\mid x_{1} \cdot y \operatorname{sgn}(x)\right\}\right)
$$

The values of $\epsilon$ is chosen based on the type of schemes, for second-order upwind scheme, $\epsilon=-1.0$. The constant $\beta$ is a compression parameter which is restricted to the range $1 \leq \beta \leq(3-\epsilon) /(1-\epsilon)$ with $\beta=6$ when $\epsilon=1$.

This process repeats for the entire discreteordinates. The nondimensional macroscopic parameters, such as velocities, temperature, and number density, are updated using the new values of $\hat{g}$ and $\dot{h}$. at each point in the physical space, based on the Modified Gaussian quadrature

$$
\begin{aligned}
& \hat{n}=\sum_{k=1}^{16} \int_{0}^{2 \pi} W_{k} \hat{g}_{k} d 0 \\
& \hat{U}_{x}=\frac{1}{\hat{n}} \sum_{k=1}^{16} \int_{0}^{2 \pi} W_{k} V_{k} \sin \circ \dot{g}_{k} d o \\
& \hat{U}_{y}=\frac{1}{\hat{n}} \sum_{k=1}^{16} \int_{0}^{2 \pi} U_{k} l_{k} \cos O g_{k} d o \\
& \dot{T}=\frac{2}{3 \hat{n}} \sum_{k=1}^{16} \int_{0}^{2 \pi} W_{k}\left(\hat{h}_{k}+V_{k}^{2} \dot{g}_{k}\right) d o-n\left(\dot{l}_{x}^{2}+\dot{l}_{y}^{2}\right)
\end{aligned}
$$

The integration over $\phi$ is obtained by the Simpson s rule. Currently, 16 points are used in o direction.

Three types of boundary conditions are implemented for the benchmark solution.

(1). For a given discrete-ordinate $l_{k}$. Reservoir Boundary Condition: 


$$
\begin{gathered}
\dot{g}_{k}=\frac{\dot{n}_{0}}{\pi \dot{T}_{0}} \epsilon^{\frac{-v_{k}^{2}}{\hat{t}_{0}}} \\
\hat{h}_{k}=\frac{1}{2} \hat{T}_{0} \hat{g}_{k}
\end{gathered}
$$

where represents the non-dimensional quantity.

(2). Symmetric Boundary Condition:

Along the nozzle and reservoir centerline, symmetric condition is applied. For a given discrete-ordinate $V_{k}$,

$$
\begin{aligned}
\left.\hat{g}_{k}\right|_{\dot{y}=0}(\pi-\phi)=\left.\hat{g}_{k}\right|_{\hat{y}=0}(\phi), & 0 \leq \phi \leq \frac{\pi}{2} \\
\left.\hat{h}_{k}\right|_{\hat{y}=0}(\pi-\phi)=\left.\hat{h}_{k}\right|_{\dot{y}=0}(\phi), & 0 \leq \phi \leq \frac{\pi}{2} \\
\left.\hat{g}_{k}\right|_{\hat{y}=0}(3 \pi-\phi)=\left.\hat{g}_{k}\right|_{\dot{y}=0}(\phi), & \pi \leq \phi \leq \frac{3 \pi}{2} \\
\left.\hat{h}_{k}\right|_{\dot{y}=0}(3 \pi-\phi)=\left.\hat{h}_{k}\right|_{\dot{y}=0}(\phi), & \pi \leq \phi \leq \frac{3 \pi}{2}
\end{aligned}
$$

(3). Wall Boundary Condition:

In order to specify the interaction between molecules and the wall surface, diffuse reflection is assumed. i.e. for a given wall temperature $\hat{T}_{w}$, and discrete-ordinate $V_{k}$, molecules which strike the surface are then emitted accordinf to a Maxwellian distribution,

$$
\begin{array}{cc}
\hat{g}_{k}=\frac{\hat{n}_{w}}{\pi \hat{T}_{w}} e^{\frac{-v_{k}^{2}}{\hat{T}_{w}}}, & \text { for }\left(\vec{V}_{k} \cdot \vec{n}\right) \geq 0 \\
\hat{h}_{k}=\frac{1}{2} \hat{T}_{w} \hat{g}_{k}, \quad \text { for }\left(\vec{V}_{k} \cdot \vec{n}\right) \geq 0
\end{array}
$$

where, $\vec{n}$ is the inward normal vector at the wall surface, Since the wall number flux $\hat{n}_{w}$ is unknown a priori, it is determined by the previous time-step value,

$\hat{n}_{w}=-2\left(\frac{\pi}{\hat{T}_{w}}\right)^{\frac{1}{2}} \int_{0}^{\infty} \int_{\phi}(\vec{V} \cdot \vec{n}) \hat{g} V d \phi d V, \quad$ for $(\vec{V} \cdot \vec{n}) \leq 0$.

Repeating above procedures, the time-marching procedure stops when convergent criteria are satisfied for all the macroscopic parameters. Currently, if the summation of relative error of density, velocity and temperature over the entire physical space is less than $10^{-5}$, the solutions are considered "converged".

\section{RESULTS AND DISCUSSION}

Numerical results of low-density nozzle flow was obtained with the developed BGK-Boltzmann-2D code. The nozzle is placed between two reservoirs which represent the upstream boundary condition and downstream boundary condition near the nozzle exit. The upstream reservoir pressure and temperature are $0.01 \mathrm{psi}$ and 900 R. The Knudsen number of the Argon gas in the chamber is 0.1 , while the Knudsen number increases to 20 at the nozzle exit. The downstream reservoir pressure is 0.0001 psi. Figure 1 shows the grid setup. Block logic is implemented in the code so that it has the ability to calculate flows with complex geometry. A more sophisticated grid network is under development and results will be addressed in the conference. The pressure ratio between the two reservoirs is selected to be 100:1 for this case. The upstream and downstream reservoir temperature is set at $900 \mathrm{R}$. The isothermal nozzle wall is set at temperature of $540 \mathrm{R}$. The Knudsen number, defined by the ratio of upstream reservoir mean free path to nozzle inlet diameter is selected to be 0.1 . Figure 2 shows the velocity vectors inside the nozzle. Figure 3 shows the Mach number contours inside the nozzle. These convergent solutions were obtained with 8 hours of CPU time on the CRAY YMP.

More extensive numerical simulations will be performed for the final presentation in the conference. As a result, a refined analysis of low-density nozzle flow characteristics will be summarized in the conference.

\section{ACKNOWLEDGEMENT}

This work is supported by NASA/MSFC research Grant NAG8-201. Mr. Lee Foster of the Structure and Dynamics Lab is the contract monitor.

\section{REFERENCES}

[1] Fisko, K.A. and Chapman, D.R., "Comparison of Burnett, Super-Burnett and Monte Carlo Solutions for Hypersonic Shock Structure," In 16th International Symposium of Rarefied Gas Dynamics," 1988.

[2] Chou, L., Deng, Z.-T. and Liaw, G.S., "Comparison of Shock Wave Structures by Solving Burnett and Boltzmann Equations," 6th AIAA/ASME Joint Thermophysics and Heat Transfer Conference, AIA A Paper 94-2056.

[3] Deng, Z.-T., Liaw, G.S. and Chou, L., "Numerical Investigation of Low-Density Nozzle Flow fields by Solving Navier-Stokes and Burnett Equations," 6th AIAA/ASME Joint Thermophysics and Heat Transfer Conference, AIAA Paper 94-2055.

[4] Cercignani, Carlo, Theory and Application of the Boltzmann Equation, Elsevier, New York, 1988.

[5] Tcheremissine, F.G., "Solution of the Boltzmann Equation for Plane Rarefied Gas Dynamic Problems." Proceedings of the 13th International Symposium on Rarefied Gas Dynamics, pp. 303-310, 1982.

[6] Tcheremissine, F.G., "Advancement of the Method of Direct Numerical Solving of the Boltzmann Equation," Rarefied Gas Dynamics: Theoretical and Computational Techniques-Progress in Astronautics and Aeronautics, Vol. 118, pp343-353, Washington, 1989, 1989, pp. 343-358.

[7] Tcheremissine, F.G., "Numerical Study of the Internal Structure of Rarefied Jets," Proceedings of the 17th International Symposium on Rarefied Gas Dynamics, pp. 299-304, Aachen, 1990.

[8] Huang, A.B., Rarefied Gas Dynamics and Plasma Laboratory Report, Georgia Institute of Technology, School of Aerospace Engineering, No. 4. 196i. 
[9] Chung. Chan Hong. Numerical Simulation of Rarcfied Gias Flow Through Nozzles and Over Submerged Bodes, Ph.D. Dissertation, University of Toledo, 1990.

[10] Hsich, K.C., "Assessment of Numerical Techniques for Cinsteady Flow Characteristics," AIA A 89-1956.

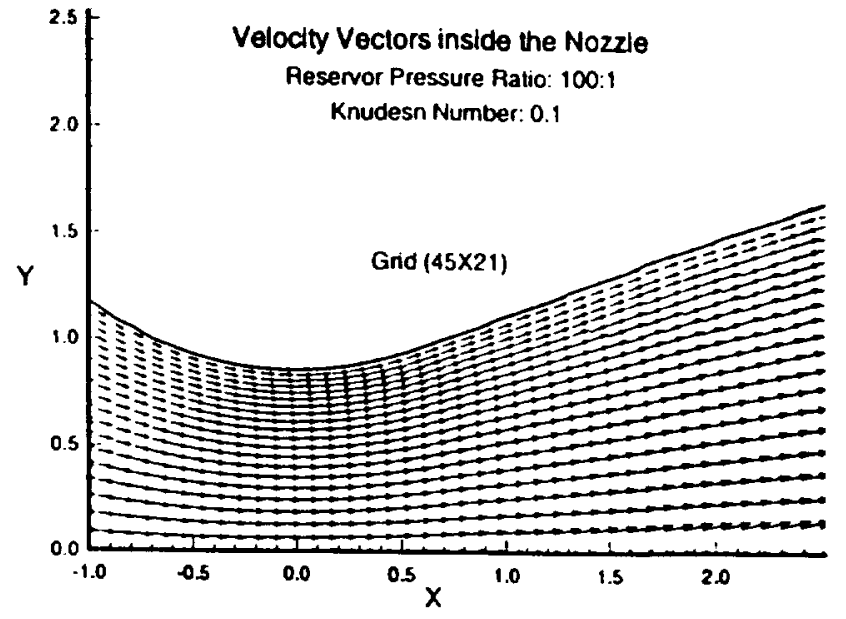

Figure 2. Velocity vectors inside the nozzle.

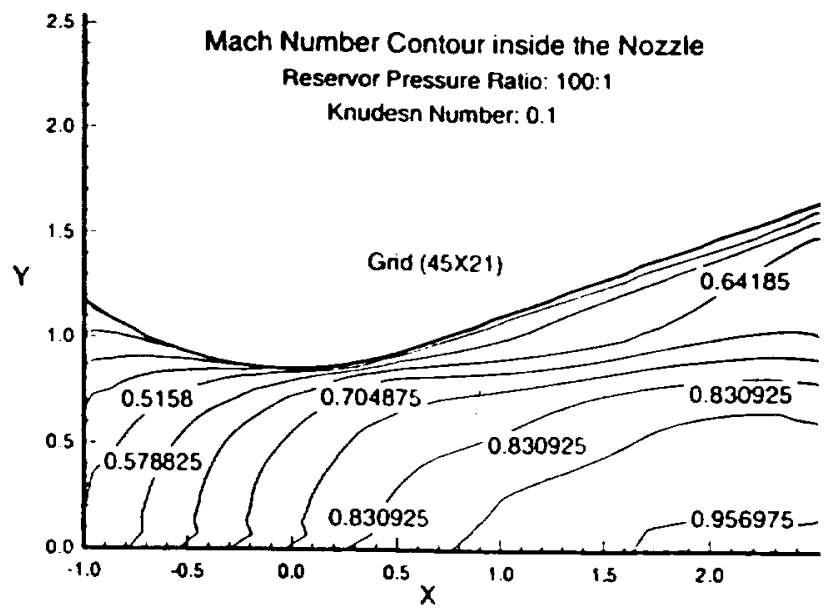

Figure 3. Mach number contours inside the nozzle. 